\title{
The responses of research participants and their next of kin to receiving feedback of genetic test results following participation in the Australian Ovarian Cancer Study
}

\author{
Nina Hallowell, DPhil ${ }^{1,2}$, Kathryn Alsop, $\mathrm{PhD}^{3,4}$, Margaret Gleeson, MSc ${ }^{5}$, Ashley Crook, MSc ${ }^{6}$, \\ Loren Plunkett, $\mathrm{MSc}^{7}$, David Bowtell, PhD $3,4,8,9$, Gillian Mitchell, PhD ${ }^{3,9}$; \\ for The Australian Ovarian Cancer Study Group ${ }^{3,10,11,12}$ and Mary-Anne Young, MSc ${ }^{3}$
}

\begin{abstract}
Purpose: The generation of clinically significant genetic data during research studies raises a number of ethical issues about the feedback of this information to research participants. Little is known about research participants' experiences of this practice.
\end{abstract}

Methods: This qualitative interview study investigated research participants' $(n=10)$ or their nominated next of kin's (relatives) $(n=15)$ experiences of receiving $B R C A 1$ and $B R C A 2$ genetic test information following participation in the Australian Ovarian Cancer Study.

Results: Interviewees had mixed responses to receiving feedback. The participants of the Australian Ovarian Cancer Study were more positive about receiving feedback, acknowledging that the genetic information may be useful for their kin. Relatives frequently described themselves as initially distressed at receiving feedback, particularly those who were unaware of the participation of their mothers in the Australian Ovarian Cancer Study. The participants of the Australian Ovarian Cancer Study and their relatives expressed an intention to disseminate the information to relatives following confirmation of the result.

Conclusion: We suggest that research participants be encouraged to discuss their participation with family members from the outset. We also outline a number of different strategies for providing feedback to research participants and their next of kin that may lessen the immediate negative impact of receiving feedback of research results.

Genet Med 2013:15(6):458-465

Key Words: consent; familial disclosure; feedback; genetics; qualitative interviews; research results

\section{INTRODUCTION}

The generation of clinically significant genetic data during research raises a number of questions about feedback, such as: should you disclose this information to the individuals concerned, and if so, what should you disclose, when, where, how, and who should you inform? ${ }^{1}$ Recent ethical debates have concluded that research participants should receive general, or aggregated, results. However, whether they should always receive individual or personal results is still debated. ${ }^{2,3}$ It is generally accepted that researchers have a responsibility to notify research participants of research findings that may significantly affect their health and/or for which preventative or therapeutic interventions are available ${ }^{4,5}$ (including intended results generated in targeted sequencing studies and incidental findings generated during whole-genome or exome-sequencing studies). Indeed, a recent review of the ethicolegal debate in this area concludes that the issue is no longer whether researchers should feed back personal information, but under what circumstances this information should be returned to research participants. ${ }^{3,6}$
Although it is agreed that the feedback of personal results to research participants, with their consent, is ethically acceptable, ${ }^{2,4,7}$ there is much less consensus about how far the researchers' duties extend, particularly, in the case of genetic/genomic research in which the information generated affects biological kin. ${ }^{4}$ Should research participants be charged with a responsibility to inform their relatives about pertinent research findings that may have an effect on their health, or should researchers contact family members? While current guidelines suggest that biological kin should have access to clinically significant research findings with their consent, ${ }^{8}$ they do not support the view that researchers have an obligation to inform relatives, ${ }^{4}$ although it has been argued that direct feedback to relatives may be ethically acceptable in rare cases (e.g., when research participants refuse to disclose information and immediate harm could be prevented). ${ }^{7,9}$

Although ethico-legal arguments favor feedback of personal genetic results, we have little knowledge about researchers' and participants' views about, or experiences of, feedback. Two recent surveys of feedback practices suggest that although 50\%

${ }^{1}$ Centre for Health and Society, University of Melbourne, Melbourne, Australia; ${ }^{2} \mathrm{PHG}$ Foundation, Strangeways Research Laboratories, Cambridge, UK; ${ }^{3}$ Peter MacCallum Cancer Centre, Melbourne, Australia; ${ }^{4}$ Department of Biochemistry and Molecular Biology, University of Melbourne, Parkville, Australia; ${ }^{5} \mathrm{Hunter}$ Genetics, Newcastle, Australia; ${ }^{6}$ Royal North Shore Hospital, Sydney, Australia; ${ }^{7}$ Murdoch Children's Research Institute, Royal Children's Hospital, Parkville, Australia; ${ }^{8}$ Department of Pathology, University of Melbourne, Parkville, Australia; ${ }^{9}$ Sir Peter MacCallum Department of Oncology, University of Melbourne, Parkville, Australia; ${ }^{10}$ Westmead Institute for Cancer Research, University of Sydney at Westmead, Sydney, Australia; ${ }^{11}$ Millennium Institute and Departments of Gynaecological Oncology, Westmead Hospital, Sydney, Australia; ${ }^{12}$ Queensland Institute for Medical Research, Brisbane, Australia. Correspondence: N. Hallowell (Nina.Hallowell@phgfoundation.org) 
of principal investigators in genetics/genomics studies ${ }^{10}$ or biobanks ${ }^{11}$ have feedback policies ${ }^{11}$ or say they had considered feeding back individualized results, ${ }^{10}$ only $~ 25 \%$ have fed back results to participants. ${ }^{10,11}$ When it comes to participants' views, research suggests that the feedback of DNA results obtained during epidemiological studies is perceived as desirable ${ }^{12,13}$ and may motivate participation. ${ }^{14,15}$ However, although (potential) research participants may be positive about receiving feedback when asked hypothetical questions, far less is known about their experiences of receiving clinically significant genetic results.

A UK interview study of patients with breast cancer, who participated in a BRCA1 and BRCA2 prevalence study, found that interviewees welcomed receiving general, or aggregate, feedback of the research findings but were much less enthusiastic about receiving personal information. Some interviewees expressed shock on learning of the increased cancer risks in their family, and others found the need for familial disclosure burdensome. ${ }^{16}$ Another study ${ }^{17}$ involving relatives of deceased patients with prostate cancer, reports that a minority experienced adjustment problems-shock, anxiety, and avoidancewhen learning about the possibility of a BRCA2 mutation segregating in the family, although these reactions were improved following genetic counseling. ${ }^{17}$ Bereaved parents who were informed that research had identified their child as suffering from a mitochondrial disorder said they were pleased that their child's biospecimens had continued to be used in the research but also expressed some concerns about the timing of feedback. Parents said they had not expected to receive feedback and that if it had occurred during the year following the death of their child, it would have been less acceptable. . $^{18,19}$

Other research suggests that individuals frequently fail to distinguish DNA testing undertaken for clinical or research purposes and commonly expect to obtain DNA test results. ${ }^{20,21}$ Ponder et al. ${ }^{20}$ for example, found that parents of children with learning difficulties who participated in the GOLD study were very grateful to finally receive feedback in the form of a genetic diagnosis of their children's impairments. The differences in the reactions of the interviewees in these studies ${ }^{16,17,20}$ may be due to the fact that the study by Ponder et al. ${ }^{20}$ involved families who had been actively seeking a (DNA) diagnosis and an etiological explanation.

To summarize, although research suggests that individuals want to receive personal research results, it appears that some are more ambivalent than anticipated when they do. Moreover, there is evidence that a proportion of research participants may be reluctant to access research results. ${ }^{22-24}$ This qualitative study sought to investigate the emotional impact on participants or their nominated next of kin of receiving feedback of research results generated during a genetic prevalence study that was carried out as a substudy of the Australian Ovarian Cancer Study (AOCS) (see Box 1).

\section{MATERIALS AND METHODS}

\section{Recruitment}

Ethical approval for this AOCS psychosocial substudy was obtained from the Peter MacCallum Cancer Centre's human

\section{Box 1: The Australian Ovarian Cancer Study}

The Australian Ovarian Cancer Study (AOCS) is a population-based study that ascertained 1,362 cases of invasive nonmucinous ovarian cancer throughout Australia between 2002 and 2006 and collected numerous biospecimens and clinical and epidemiological data. In January 2009, DNA testing began to determine the prevalence of BRCA1 and $B R C A 2$ mutations on a subsample of 1,001 women (AOCS prevalence substudy). ${ }^{34}$

On recruitment to AOCS, at the time of their ovarian cancer diagnosis, all participants were asked whether they would like any research results of potential personal clinical significance (including potential genetic findings) to be made available to themselves and their family. Ninety-seven percent $(971 / 1,001)$ of those women whose DNA samples were subsequently used in the prevalence substudy had opted for personal feedback of research results upon entry to AOCS, and $94 \%(945 / 1,001)$ had also given permission for their results to be made available to family members in the event that they were unable to receive the results themselves. When participants consented to the study, the AOCS researchers had also requested details of a contact person to facilitate recontact with the participant in case they were lost to follow-up. Contacts were provided by $80 \%(800 / 1,001)$ of women used in the prevalence substudy, including daughter $(35.5 \%)$, son (14\%), parent (5\%), partner (12\%), other family ( $21 \%)$, or friend $(12 \%)$. Only $76 \%$ (757) women who had indicated they would like family members to receive feedback of clinically significant information provided the study with contact details of a next of kin, or an alternative contact that could be used in the case of researchers losing contact with the participant. The nominated contacts/next of kin were not required to acknowledge their nomination.

Between April 2009 and July 2011, AOCS participants in whom a $B R C A$ mutation was identified, or their nominated next of kin if the woman was deceased, were notified in writing (Supplementary Appendix online) by the AOCS researchers, as follows:

"...Our research has identified information relevant to your family. This means that a genetic change has been found in your family which may account for the family's experience of cancer" (AOCS notification letter, October 2007, original emphasis).

The letter explained the nature of research testing, the need for confirmation in a clinical (quality assured) laboratory and encouraged recipients to seek further information from a family cancer clinic.

\begin{abstract}
"We strongly recommend that you discuss this letter with a genetic counselor or doctor at a family cancer clinic. This information may be very important for you or for other family members in reducing the risk of cancer" (AOCS notification letter, October 2007, original emphasis).
\end{abstract}

A list of contact details for the different family cancer clinics in Australia was included. 
research ethics committee. Inclusion criteria included being an AOCS participant (or nominated next of kin), receiving a notification letter about genetic research results, age $>18$ years, and capable of speaking in English.

All eligible interviewees were sent an invitation to participate in an interview with an opt-out card. A researcher telephoned the recipient to arrange an interview 2 weeks later. In accordance with the practice in qualitative research, interview analysis was ongoing during the period of data collection; this dictated the sampling strategy, and recruitment ceased when new themes failed to emerge in the data set.

\section{Data collection and analysis}

In-depth semistructured telephone interviews (30-90 min) were conducted from May 2010 to September 2011. Interviews were informed by a topic guide, in which emerging themes in earlier interviews became the focus of targeted questioning in subsequent interviews. The following themes were explored: recollection of receiving the notification letter, understanding and responses to the letter, family communication about the letter, and factors influencing decision making about follow-up in the familial cancer clinic.

Interviews were tape-recorded with consent and transcribed. Data analysis was conducted using an inductive approach, and transcripts were coded using the method of constant comparison. ${ }^{25}$ This is an iterative process that involves systematically identifying, comparing, and coding themes within and between interviews. Emerging patterns and relationships between the codes led to the development of second-order categories (e.g., emotional responses, patterns of disclosure). Deviant cases were taken into consideration in the analysis. Analytical rigor was achieved by multiple research team members coding interviews to confirm themes.

\section{RESULTS}

A total of 25 individuals participated in the psychosocial substudy: 10 AOCS participants (AOCS) and 15 next of kin (relatives). The number of weeks between receiving the notification letter and the interview varied widely in both groups (AOCS: mean 19, range 2-46; relatives: mean 14, range 4-45) (Table 1).

This study focuses on interviewees' accounts of their emotional reactions to the letter and their (intended) disclosure practices. Identifying information has been removed and pseudonyms are used throughout.

\section{Emotional responses to feedback}

Interviewees described their feelings upon receiving the notification letter using a range of emotional terms. Some were positive ("excited," "pleased"), some negative ("angry," "scary," "freaked me out a little"), and others more neutral ("ambivalent," "not surprised").

Emotional responses to feedback: AOCS. Some AOCS participants said they were pleased to receive the letter because it legitimated previous concerns about relatives' risks. Nellie
Table 1 Interviewees' demographic characteristics

\begin{tabular}{|c|c|c|c|c|}
\hline & \multicolumn{2}{|c|}{ AOCS } & \multicolumn{2}{|c|}{ Relatives } \\
\hline & Range & Mean & Range & Mean \\
\hline Age (years) & $53-80$ & 65 & $28-62$ & 42 \\
\hline \multirow[t]{2}{*}{ Years since diagnosis } & $5-8$ & 6 & & \\
\hline & & $n=10$ & & $n=15$ \\
\hline \multicolumn{5}{|l|}{ Relatives' relationship to AOCS } \\
\hline Daughters & & - & & 12 \\
\hline Husband/partner & & - & & 2 \\
\hline Daughter-in-law & & - & & 1 \\
\hline \multicolumn{5}{|l|}{ Educational level } \\
\hline No postschool qualifications & & 2 & & 4 \\
\hline Postschool qualifications & & 8 & & 11 \\
\hline \multicolumn{5}{|l|}{ Marital status } \\
\hline Married or living as married & & 7 & & 11 \\
\hline Divorced or separated & & 2 & & 2 \\
\hline Widowed or never married & & 1 & & 2 \\
\hline Children & & 8 & & 13 \\
\hline Number with girls & & 6 & & 10 \\
\hline Number with boys & & 7 & & 7 \\
\hline
\end{tabular}

AOCS, Australian Ovarian Cancer Study.

(AOCS) said that when she received the letter she thought "Oh right, now we're starting to do something." These women understood that research participation had generated useful information for their family. As Margaret (AOCS) said: "I was probably quite pleased in a way because they realized there was some connection there and that will help my family."

Although most AOCS described their first reaction using positive terms, a couple reported feeling angry or annoyed when they first read the letter, primarily because its contents brought back memories of their illness and cancer treatment.

I was really annoyed, annoyed that something else had... and I just got fed up with thinking about it [cancer] and wished you could put it to the side...you come to the feeling when is it all going to end. (Debbie, AOCS).

Emotional responses to feedback: relatives. In contrast to the majority of AOCS participants, relatives frequently described themselves as initially experiencing a range of negative emotions upon receiving the letter. Some said that it had upset them:

Interviewer: What was your immediate response when you read the letter?

Sarah: Shit... I was just talking to my husband, it's just that letter on genetic things. Oh yeah, a little bit, bit upset (Sarah, daughter).

Others, particularly biological relatives, who were potentially at risk of cancer, perceived the letter and its implications as confronting or frightening. 
I found it [the letter] a bit confronting and a bit scary... and there's that bit in bold....and it said it that a genetic change has been found in your family....I mean it's a bit of a confronting thing and maybe I'm hesitant in finding out the results in case they say you know there's a big genetic link or something (Helen, daughter).

Indeed, for some relatives this information was regarded as very distressing. These women were ambivalent about receiving the notification letter, focusing on the negative consequences of knowing and living with risk. They said that they were not sure that they wanted to know this information about themselves, and talked about how they needed some time to come to terms with receiving the letter and acting on it. Kate (daughter) described the letter as "distressing" and said that she "didn't really want to know that." Likewise, Sarah (daughter) said: "I don't really want to know if I've got it. If I'm going to get it... I'm still in two minds whether I want to know if I've got it or not."

Other relatives said they were shocked to receive the notification letter. In three cases, this was because they were unaware that their mother had participated in AOCS, as Helen (daughter) said, "I had no idea she was in any sort of study. So it was a bit of a shock." The majority of relatives (8/15) said they had known of their family member's participation in AOCS, but thought it was a questionnaire survey and were unaware that it involved genetic testing and therefore had direct implications for their health. A third (5/15) of the relatives said they were shocked to receive feedback so long after their parent or relative had died.

It's always a shock to find out there is a possibility that you could have inherited a genetic change... it is a shock also because it's been you know, 4 years since my Mum died and you think, well, why did it take so long? (Mabel, daughter).

\section{Ambivalent responses to feedback}

However, most interviewees, both AOCS and relatives, talked about experiencing a range of mixed emotions; describing the contents of the letter as "frightening," "distressing," "scary," while acknowledging that this information could help self and/ or other family members.

When I read the letter it was like oh this is a bit scary...but I was also relieved as well to know I could go and get a test.... In talking with my sister and my auntie, you just went, "how lucky we are that weve got this opportunity to go and see if we are genetically disposed and deal with it?" (Emma, daughter).

Thus, receiving the notification letter was perceived by many interviewees as a double-edged sword.

I like the information, I like knowing all about it, I like anything that might do any good, but when you find out you've got a genetic link, you think, "oh bastard, there's nothing you can do" (Debbie, AOCS).

These ambivalent responses were in part due to the recognition that the information contained in the notification letter has different implications for self and others.

\section{Relevance of information for self and others}

Some AOCS participants did not regard receiving feedback as having any implications for their health because they had already had ovarian cancer.

It was just irrelevant because I don't have any children. I don't really think that knowing if I've got the gene now at my stage of life is of any importance... Nothing good is going to come out of it for me (Bertha, AOCS).

However, although many of the AOCS participants observed that the information contained in the letter had little relevance for themselves, they acknowledged that it might be important for others, and felt they had a responsibility to follow it up for their relatives.

I'm probably pretty blasé about it [cancer]. If it's going to happen, it's going to happen.... and didn't really want to follow it up so much. Then when my daughter said about it, I thought, "oh, I suppose for the kids' sake, I really should" (Irene AOCS).

Others, particularly relatives, talked about how everyone, themselves and their family, could potentially benefit from receiving this information.

I mean, sometimes it's scary but it's nice to have the information and, and certainly, another way of looking at it, is it helps other people I'd like to know for myself but it's more for my daughter, ... if there is a possibility, then we need to be more vigilant...forearmed is forewarned (Kerry, daughter).

In summary, many interviewees were ambivalent about receiving feedback, regarding it as irrelevant or anxiety provoking. However, although these interviewees may have preferred not to engage with this information, they recognized that their relatives could benefit from receiving it and they proceeded, or intended, to disclose it to family members.

\section{Disclosing research findings to family}

With a couple of exceptions, most interviewees reported mentioning the feedback letter to at least one other person.

When I got the letter...I just got on the phone and called my sister and said, "we've got to get a test"... and my auntie, "you've got to get a test"... (Emma, daughter). 
In most cases, this was a partner, sibling, or child, and disclosure was justified on the basis of other people's right to access this information. For example, Andrew (husband) made it clear he had a duty to inform his wife's family about the AOCS results, and so he had told one of her sisters and intended to tell others.

I think now that I have this information, I have to make very reasonable effort to communicate that to those people [wife's family].

Even those who found disclosure difficult because of disrupted relationships said they had made an effort to inform relatives. For example, Ruth (AOCS) asked her former daughter-in-law to relay the information to her estranged daughter. She had also told her grandchildren and other family overseas. She described the poor reception the news had received, but concluded that despite the fact that some people "...don't want to be told, you have to tell them."

Only one interviewee, Bertha (AOCS), argued that perhaps ignorance is bliss, and that her nieces did not automatically have the right to this information and, even if they did, she was not sure that she should disclose it.

Knowing you've got it, is not necessarily a good thing, because you live in that shadow. But why do they need to know, they deserve to have their lives free like I had mine until 42 ...I mean that's where their heads are at. Who am I to burst their bubble? I'm just an auntie.

The difficulties hinted at by Bertha were echoed by others who said that they were delaying informing certain relatives. In some cases, they had already discussed the feedback letter with some family members, but were going to wait until the result was confirmed before talking to others. As Julie (AOCS) said about talking to her brother, "until such time that I have any results, there's actually no point discussing it with anyone." In most cases, the delay was justified to avoid causing unnecessary anxiety.

I [won't talk to] my mother-in-law just because I know that she worries, so she doesn't need the extra extra stress of what it could entail until I know for sure that there is a possibility, then she doesn't need to know (Kerry, daughter).

\section{DISCUSSION}

This study suggests that research participants and next of kin have subtly different reactions to receiving personal feedback of clinically significant research findings. Although a minority of AOCS participants were initially distressed at receiving the notification letter, most were positive about receiving feedback because they thought it might help other family members manage their cancer risks. Indeed, nearly all AOCS participants either had, or said that they would, discuss the letter with relatives at an appropriate time. Although relatives were similarly prepared to disclose genetic information to their family, they were more ambivalent about receiving feedback. Many biologically related relatives reported initially feeling threatened or distressed upon receiving the news that they may be at increased risk of ovarian cancer.

When interpreting these data, we need to be mindful of the fact that AOCS included a population-based sample, which meant that in many interviewees' families there was no strong history of ovarian cancer and, therefore, no culture of living with familial risk, which is typically reported in studies of highrisk women. Thus, for many relatives, the information in the notification letter came as a "bolt out of the blue," particularly, as some were completely unaware that (i) their family member had participated in AOCS, (ii) AOCS could reveal genetic results, and/or (iii) personalized feedback of AOCS results could arrive years after their relative's death. So can we draw any conclusions from these data about the practice of feeding back research findings to participants and family members?

Most AOCS participants experienced receiving personal feedback as a positive event, not least because they recognized that their concerns about their family were being taken seriously. However, although AOCS, like those who attend genetic counseling, are mindful of the familial implications of genetic information, ${ }^{26}$ a minority was distressed to receive this information because it reminded them of their cancer and its treatment. For some who felt they were finally putting their illness behind them, receiving information about their genetic risk status was experienced as upsetting. Similar findings have been reported in patients with cancer who have undergone $B R C A$ testing who describe finding out about their genetic risk as destabilizing their identity management. ${ }^{27}$ Clearly, we need to recognize that for some research participants, particularly those undergoing or coming to terms with treatment, the timing of feedback is crucial. ${ }^{18,19}$ We need to be wary of overloading individuals with emotionally laden information at critical times in their illness trajectory. It is of course difficult for researchers to judge the appropriateness of the timing of feedback in particular cases; one way of overcoming this problem would be to let research participants choose when to access personalized feedback.

It is generally agreed that researchers have an obligation to notify research participants of clinically significant information, but there are questions about how far this obligation extends. ${ }^{4}$ Because the AOCS prevalence substudy involved a population who had a strong chance of dying before the release of any meaningful genetic data, it was anticipated that a significant number of nonparticipating relatives (individuals who did not stand in an explicit relationship to researchers) would receive notification of the research findings. Upon recruitment to AOCS, study participants were asked if they wanted next of kin to receive their results from researchers; however, consent for this feedback was not required from these relatives. Our data suggest that whereas the majority of relatives knew of their relatives' participation in the main AOCS study, they were not aware of the prevalence substudy, and therefore had 
not anticipated receiving genetic results and were shocked to receive feedback.

There are steps we could take to avoid relatives receiving unanticipated genetic test results. First, we could require, or at least try to obtain, consent from relatives to receive feedback at the outset, particularly in studies like AOCS, in which there is a possibility that research results may be generated in the future and/or the research participants may die before their release. ${ }^{4,7,9}$ It has been argued that as genetic research potentially reveals results about other family members, to protect individuals privacy rights, a more family-centered approach should be adopted as a matter of course. ${ }^{9}$ McGuire et al..$^{9}$ argue that although there can be no legal requirement to obtain consent from biological relatives before an individual participates in genetic research, potential research participants should be encouraged to involve other family members in the decision-making process. Following these authors, we suggest that in cases for which it is not practical to obtain relatives' written consent for feedback in advance, research participants should be strongly encouraged to inform their kin about their participation, the possibilities of data release and/or feedback, and the implications for their privacy. In addition to involving at-risk relatives (e.g., adult children and siblings), we suggest that research participants also inform their spouses/partners. Although this group may be only indirectly implicated in feedback, by caring for the research participant or parenting at-risk offspring, they could act as a conduit for familial disclosure, as was the case of the married-in kin who received feedback in this study.

Although the adoption of a family-based approach to research participation may enable us to circumvent the problem of unanticipated feedback to next of kin, it does not solve it, for it is possible that genetic results may be generated many decades after research participants and their consented/informed next of kin are deceased. What happens then? Do researchers have an obligation to approach research participants' (nonconsented) descendants many years into the future, and, if so, when will these duties and the duties of future researchers, be discharged? Moreover, identifying and tracing research participants' descendants raises a number of practical questions, such as who they are and how to find them? Although those who work in genetic clinics are proficient at family tracing, epidemiological researchers may not have the same degree of expertise or resources.

One way to avoid these problems is to not only regard genetic research results as a family resource ${ }^{28}$ but also to feed back research results in a staggered, or multistep way to research participants and their family. The process could begin with individuals who consent to feedback being informed that aggregated (generic) results of the study are available and that there are potential opportunities for them to obtain more personalized findings that may/may not have implications for their family; they could then be given instructions about how and where they and their relatives can retrieve this information. This process could be supported by the provision of access to genetic counseling ${ }^{17}$ for distressed participants or relatives who are concerned about the personal implications and require assistance with decision making about accessing more personalized information and communicating it within their family.

This feedback process could be enhanced by the use of Webbased interactive information technology that would provide ongoing access to research information for research participants and family members, in the present or the future. Webbased forms of information delivery would allow research participants and/or relatives to access more or less personal feedback as and when they want it, thereby enabling them to decide exactly what information they obtain. ${ }^{29}$ In other words, the use of interactive information technology would allow for the personalization of feedback by enabling recipients to dictate its scope. Although the delivery of staggered feedback may be complex to initiate, it could help researchers fulfill their obligations to provide research participants (and relatives) with feedback, while also allowing individuals to preserve their right to not know personal information about themselves (see also National Health and Medical Research Council of Australia guidelines). ${ }^{8}$ Clearly, problems could arise if some family members want to access more personalized information than others, but these situations frequently occur in clinical genetics and are resolved through genetic counseling. Furthermore, the argument that individuals' access to genetic information about themselves potentially threatens other family members' genetic privacy, 9,30 may become less relevant once exome and whole-genome sequencing becomes a more widespread clinical practice.

Although staggering feedback has the potential to preserve individual autonomy and genetic privacy, it is not without problems. First, it has been argued that the adoption of participant-centric initiatives such as this requires a shift in the way in which we conceive of patients and research participants; we need to see them as responsible, active research collaborators rather than passive contributors of clinical/research samples/ information. ${ }^{29}$ Second, it requires researchers to monitor and update the available information and information delivery platforms; this is labor intensive and, therefore, costly. Third, if feedback relies on certain types of interactive information technology, then only those who have access to these forms of technology may be able to access this information, and this is a potential source of inequality. ${ }^{29}$ Finally, although staggered feedback (plus/minus interactive information technology) provides research participants (and relatives) with an active role in the feedback process, it still relies on research participants informing relatives that they have participated in research; that the research may have implications for the wider family; and that relatives can find out about it by reading a newsletter or accessing a particular website. Also, as the AOCS prevalence substudy indicates, research participants may not be very good at communicating this information up-front.

Despite the above arguments, framing of genomics research as a family-centered enterprise and encouraging family communication about research involvement from the outset provides a practical solution to the ethical debate 
about feedback to family members. Moreover, this familycentered approach reflects the views of those who received feedback in this study. Our data indicate that interviewees were aware of the familial ramifications of genetic epidemiological research and regarded the familial disclosure of research results as a priority. These findings echo those of Wakefield et al., ${ }^{24}$ who found that many kConfab participants who had received feedback cited a need to obtain information for relatives as a primary motivation for attending an family cancer clinic or having $B R C A$ genetic testing. The author ${ }^{24}$ note that interviewees who failed to follow up feedback letters described the information as not personally relevant or had a different understanding of its value. The idea that failure to follow up feedback and disclose research results to family is not motivated by any malicious intent, is confirmed by an international study that suggested that only $0.09 \%(9 / 9,914)$ of patients attending genetic counseling for hereditary cancers stated that they would not inform their relatives when encouraged to do so, and in most of these cases, (intended) nondisclosure was motivated by beneficent reasons, such as not wanting to cause anxiety to others. ${ }^{31}$ Thus, it would seem that research participants are happy to disclose information to their relatives; perhaps what is needed is more emphasis on the potential familial implications of participation in genetic/genomics research.

Before concluding, it is important to acknowledge the limitations of this study. Although no consistent differences emerged in the responses of individuals interviewed at different times since receiving feedback, we should recognize that the lack of standardized times between the letter arriving and the interview may have meant that some accounts were subject to recall bias. We must also note that our method of recruitment meant that we only accessed people who wanted to talk about receiving feedback and its implications. This reflects a common problem faced by researchers, namely, how to represent the views of those who do not want to engage with genetic services ${ }^{32}$ or researchers. ${ }^{22,24}$ What these people feel, understand, need, or go on to do, we do not know.

We note that the small number of married-in relatives recruited to this study meant we were unable to draw meaningful conclusions about differences between their responses and those of biologically related relatives. Future research should aim to systematically compare the impact of feedback on relatives who are directly and indirectly affected by/implicated in the feedback of genetic information.

\section{Conclusion}

Despite the long-running ethico-legal debate concerning the rights and wrongs of feeding back individualized research results, there is little empirical evidence about the impact of this practice on research participants and their kin. This study provides some data to fill that gap. It suggests that receiving feedback generates complex and often contradictory emotional responses in research participants and their next of kin. However, these data are only a start, for when interpreting these findings we must be mindful of the fact that the feedback process in the AOCS prevalence substudy was (relatively) straightforward. The AOCS results were single, anticipated, pertinent, nonincidental findings, and the research participants, at least, had consented (i.e., wanted) to receive them. Yet despite this relatively simple feedback scenario, receiving research results generated a range of complex emotional responses in our interviewees and a range of ethical issues for researchers who were managing this process. Current debate about the provision of personalized feedback following genomics research focuses on the feedback of the results of whole-genome- or exomesequencing studies. ${ }^{6,33}$ These studies have the potential to produce a range of anticipated and unanticipated (incidental/ coincidental) findings, which have differing health and social implications, a number of varying risks, and a range of certainties and uncertainties associated with them. Feeding back such results will be a much greater challenge than that posed by the AOCS prevalence substudy. Indeed, despite ongoing debate about who, where, when, and how we feed back such information, we really have very little clear idea about what we could, or, perhaps more importantly, should feed back to research participants and their kin and how they will take it. ${ }^{33}$ Will it cause harm or will it be of ultimate personal and public benefit? We do not know the answer to this question.

\section{SUPPLEMENTARY MATERIAL}

Supplementary material is linked to the online version of the paper at http://www.nature.com/gim

\section{ACKNOWLEDGMENTS}

We thank all the AOCS participants, their relatives who took part in these interviews, and all the women who took part in the Australian Ovarian Cancer Study (AOCS). N.H. thanks the Centre for Health and Society at the University of Melbourne, kConfab, which accommodated and hosted her visit to Australia in 2011, and the Institute of Health \& Society, Newcastle University, which granted her study leave. She also thanks Martin Richards for the interesting discussions about feedback. The AOCS was approved by the Human Research Ethics Committees at the Peter MacCallum Cancer Centre, Queensland Institute of Medical Research, University of Melbourne, New South Wales Cancer Council, the Cancer Council South Australia, the Cancer Foundation of Western Australia, and all participating hospitals. AOCS was supported by the US Army Medical Research and Materiel Command under DAMD17-01-1-0729, the Cancer Council Victoria, Queensland Cancer Fund, the Cancer Council New South Wales, the Cancer Council South Australia, the Cancer Foundation of Western Australia, the Cancer Council Tasmania, and the National Health and Medical Research Council of Australia (NHMRC; ID400413, ID40028). The entire study group can be found at http://www. aocstudy.org. The AOCS prevalence study was supported by the Ovarian Cancer Research Program of the US Department of Defense (W81XWH-08-1-0684 and W81XWH-08-1-0685), Cancer Australia (509303), and the Peter MacCallum Cancer Centre Foundation. We gratefully acknowledge the cooperation of the participating institutions in Australia, and also acknowledge the 
contribution of the study nurses, research assistants, and all clinical and scientific collaborators. Finally, we thank Sam Wake for her help with this interview study.

\section{DISCLOSURE}

The authors declare no conflict of interest.

\section{REFERENCES}

1. Clayton EW, McGuire AL. The legal risks of returning results of genomics research. Genet Med 2012;14:473-477.

2. Affleck P. Is it ethical to deny genetic research participants individualised results? J Med Ethics 2009;35:209-213.

3. Bredenoord AL, Kroes HY, Cuppen E, Parker M, van Delden JJ. Disclosure of individual genetic data to research participants: the debate reconsidered. Trends Genet 2011;27:41-47.

4. Knoppers BM, Joly Y, Simard J, Durocher F. The emergence of an ethical duty to disclose genetic research results: international perspectives. Eur $J$ Hum Genet 2006:14:1170-1178.

5. Wolf SM, Lawrenz FP, Nelson CA, et al. Managing incidental findings in human subjects research: analysis and recommendations. J Law Med Ethics 2008;36:219-48, 211.

6. Wolf $\mathrm{SM}, \mathrm{Crock} B N$, Van Ness B, et al. Managing incidental findings and research results in genomic research involving biobanks and archived data sets. Genet Med 2012;14:361-384.

7. Kollek R, Petersen I. Disclosure of individual research results in clinico-genomic trials: challenges, classification and criteria for decision-making. J Med Ethics 2011;37:271-275.

8. NHMRC. National Statement of Conduct in Human Research. 2007. http:// www.nhmrc.gov.au/book/chapter-3-5-human-genetics Accessed 6 May 2012.

9. McGuire AL, Caulfield T, Cho MK. Research ethics and the challenge of wholegenome sequencing. Nat Rev Genet 2008;9:152-156.

10. Heaney C, Tindall G, Lucas J, Haga SB. Researcher practices on returning genetic research results. Genet Test Mol Biomarkers 2010;14:821-827.

11. Johnson $G$, Lawrenz $F$, Thao $M$. An empirical examination of the management of return of individual research results and incidental findings in genomic biobanks. Genet Med 2012;14:444-450.

12. Murphy J, Scott J, Kaufman D, Geller G, LeRoy L, Hudson K. Public expectations for return of results from large-cohort genetic research. Am J Bioeth 2008;8:36-43.

13. Opinion Leader. Assessing public attitudes to health related findings in research. Wellcome Trust and Medical Research Council: London, 2012

14. Wendel D. The debate over research on stored biological samples. Arch Intern Med 2002;162,1457-1462.

15. Gustafsson Stolt U, Ludvigsson J, Liss PE, Svensson T. Bioethical theory and practice in genetic screening for type 1 diabetes. Med Health Care Philos 2003;6:45-50.

16. Richards MP, Ponder M, Pharoah P, Everest S, Mackay J. Issues of consent and feedback in a genetic epidemiological study of women with breast cancer. $J$ Med Ethics 2003;29:93-96.
17. Ormondroyd E, Moynihan C, Watson M, et al. Disclosure of genetics research results after the death of the patient participant: a qualitative study of the impact on relatives. J Genet Couns 2007;16:527-538.

18. Sexton AC, Sahhar M, Thorburn DR, Metcalfe SA. Impact of a genetic diagnosis of a mitochondrial disorder 5-17 years after the death of an affected child. $J$ Genet Couns 2008;17:261-273.

19. Sexton AC, Metcalfe SA. Disclosing genetic research results after death of pediatric patients. JAMA 2008;300:1693-1695.

20. Ponder M, Statham H, Hallowell N, Moon JA, Richards M, Raymond FL. Genetic research on rare familial disorders: consent and the blurred boundaries between clinical service and research. J Med Ethics 2008;34:690-694.

21. Cooke S, Crawford G, Parker M, Lucassen A, Hallowell N. Recall of participation in cancer genetics research projects. Clin Ethics 2008;3:180-184.

22. Keogh LA, Southey MC, Maskiell J, et al. Uptake of offer to receive genetic information about BRCA1 and BRCA2 mutations in an Australian population-based study. Cancer Epidemiol Biomarkers Prev 2004;13:22582263.

23. Treloar SA, Morley KI, Taylor SD, Hall WD. Why do they do it? A pilot study towards understanding participant motivation and experience in a large genetic epidemiological study of endometriosis. Community Genet 2007;10:61-71.

24. Wakefield C, Ratanayake P, Meiser B, et al. For all my family's sake, I should go and find out. An Australian Report on Genetic Counseling and Testing Uptake in Individuals at High Risk of Breast and or Ovarian cancer. Gen Testing MolBiomarkers 2011;15:397-385.

25. Strauss A, Corbin J. Basics of Qualitative Research. Sage: London 1991.

26. Hallowell N, Foster C, Eeles R, Ardern-Jones A, Murday V, Watson M. Balancing autonomy and responsibility: the ethics of generating and disclosing genetic information. J Med Ethics 2003;29:74-9; discussion 80.

27. Hallowell N, Foster C, Eeles R, Ardern-Jones A, Watson M. Accommodating risk: responses to BRCA1/2 genetic testing of women who have had cancer. Soc SCi Med 2004;59:553-565.

28. Parker M, Lucassen AM. Genetic information: a joint account? BMJ 2004;329:165-167.

29. Kaye J, Curren L, Anderson N, et al. From patients to partners: participant centric initiatives in biomedical research. Nat Rev Genet 2012;13:371-376.

30. Laurie GT. In defence of ignorance: genetic information and the right not to know. Eur J Health Law 1999;6:119-132.

31. Clarke A, Richards M, Kerzin-Storrar L, et al. Genetic professionals' reports of nondisclosure of genetic risk information within families. Eur J Hum Genet 2005; 13:556-562.

32. Suthers GK, Armstrong J, McCormack J, Trott D. Letting the family know: balancing ethics and effectiveness when notifying relatives about genetic testing for a familial disorder. J Med Genet 2006;43:665-670.

33. Knoppers BM, Kharaboyan L. "Deconstructing" biobank communication of results. Scripted 2009;6:677-684.

34. Alsop K, Fereday S, Meldrum C, et al. BRCA mutation frequency and patterns of treatment response in BRCA mutation-positive women with ovarian cancer: a report from the Australian Ovarian Cancer Study Group. J Clin Oncol 2012:30:2654-2663. 